\title{
Alimentação Saudável no Instagram: Rede de Hashtags
}

\author{
Júlia Carvalho Andrade, Núbia Moura Ribeiro', Eneida Baumann¹, Vanda Lima \\ Maltez do Espírito Santo ${ }^{2}$, Hernane Borges de Barros Pereira ${ }^{1,3}$ \\ ${ }^{1}$ Doutorado Multi-Institucional e Multidisciplinar em Difusão do Conhecimento - \\ Universidade Federal da Bahia (UFBA) - Salvador - BA - Brasil \\ ${ }^{2}$ Centro Universitário Maurício de Nassau (UNINASSAU) - Salvador - BA - Brasil \\ ${ }^{3}$ Programa de Pós-Graduação em Modelagem Computacional e Tecnologia Industrial \\ (SENAI-CIMATEC) - Salvador - BA - Brasil \\ juliacarvalhoandrade@yahoo.com.br, feneidabaumann, nubiamouraribeiro, \\ vandamaltez2, hbbpereira\} @gmail.com
}

\begin{abstract}
Nowadays, social media has been widely used to search and share information about healthy eating. The goal of this study was to analyze the representation of the term 'healthy eating' in Instagram by means of a hashtags network based on cliques. The study was conducted in seven steps. The most important hashtags in terms of degree were \#saude, \#dieta, \#fitness, \#vidasaudavel, \#comidadeverdade, \#emagrecer, \#emagrecimento, \#nutrição, \#saúde and \#reeducaçãoalimentar. We analyzed 10 main communities, of which five relate to the biological aspect of adequate and healthy eating, while the others also highlight cultural, environmental and hedonic aspects.
\end{abstract}

Resumo. Atualmente, as mídias sociais têm sido muito utilizadas para buscar e compartilhar informações sobre alimentação saudável. O objetivo deste estudo foi analisar a representação do termo "alimentação saudável" no Instagram por meio de uma rede de hashtags de cliques. O estudo foi conduzido em sete etapas. As hashtags mais importantes em termos de grau foram \#saude, \#dieta, \#fitness, \#vidasaudavel, \#comidadeverdade, \#emagrecer, \#emagrecimento, \#nutrição, \#saúde e \#reeducaçãoalimentar. E analisamos as 10 principais comunidades, das quais cinco se relacionavam com o aspecto biológico da alimentação adequada e saudável, enquanto as outras também ressaltaram aspectos culturais, ambientais e hedônicos.

\section{Introdução}

As recomendações para promoção da alimentação adequada e saudável reforçam o direito humano básico de acesso permanente e regular à alimentação. Segundo o Guia Alimentar para a População Brasileira, a alimentação adequada e saudável deve ser baseada em uma variedade de alimentos que contemple as necessidades biológicas, sociais e emocionais dos indivíduos, além de necessidades alimentares especiais; deve ser justa do ponto de vista social; ter como referência a cultura alimentar e as dimensões de gênero, raça e etnia; ser acessível física e financeiramente; equilibrada, harmônica e moderada em quantidade e qualidade; prazerosa; e sustentável [Brasil 2014]. 
Para Kuwae et al. (2013) é no interior das relações sociais que surge a definição do que é bom para comer e consumir, segundo a cultura alimentar e as influências econômicas, midiáticas, estéticas, morais, higiênicas, entre outras, que modelam o comer. Assim, por conta da ampliação das relações sociais pelas mídias sociais, nestes espaços também ocorre a (re)construção do que seria uma boa alimentação. Por isso, torna-se relevante analisar o grande volume de informações sobre alimentação saudável difundidas rapidamente e constantemente nas mídias sociais.

Segundo a Pesquisa Brasileira de Mídia 2016 [Brasil 2017], atualmente, 49\% dos brasileiros utilizam a Internet como primeira ou segunda fonte de informação e o tempo dedicado à Internet é maior que às mídias tradicionais. Ainda segundo essa pesquisa, as mídias sociais mais utilizadas no Brasil em ordem decrescente são YouTube, Facebook, WhatsApp, Messenger, Instagram, Google+e Twitter.

A análise de conteúdo nas mídias sociais implica na análise dos elementos das postagens: perfil dos usuários, imagens, textos, compartilhamentos, comentários, "curtidas" e hashtags ou apenas tags. As hashtags são termos associados a uma informação, tópico ou discussão, representadas pelo símbolo cerquilha (\#), por exemplo, \#food. As hashtags se referem ao conteúdo postado e permitem a catalogação das postagens, recuperação de informações e representação do conhecimento.

Neste estudo interdisciplinar sobre mídias sociais e alimentação, utilizamos a teoria e ciência de redes para investigar se por meio de uma rede de hashtags de cliques é possível analisar como o tema alimentação saudável tem sido representado em uma mídia social, e se está em consonância com o conceito de alimentação adequada e saudável do Guia Alimentar para a População Brasileira [Brasil 2014].

Escolhemos como cenário a mídia social Instagram por ser muito utilizada para compartilhar fotos de comidas e ser popular nas áreas de alimentação e nutrição. Essa plataforma está disponível em smartphones e computadores e possui mais de um bilhão de usuários ativos em todo o mundo [AFP 2018]. Ademais, o Instagram permite a manipulação estética de imagens, vinculação de textos às imagens, comentar e "curtir" as postagens dos usuários por meio de um botão de coração. Também possibilita a classificação de postagens utilizando até 30 hashtags distintas. O sistema de tagging do Instagram possibilita a divulgação dos mais diversos conteúdos, a catalogação das postagens dentro de determinada temática e a busca de imagens que foram marcadas com determinadas hashtags [Gauziski e Maia 2013].

O objetivo deste estudo é analisar a representação do termo "alimentação saudável" por usuários produtores e consumidores de conteúdo no Instagram e verificar se essa representação está em consonância com o conceito de alimentação adequada e saudável [Brasil 2014] por meio das hashtags utilizadas pelos usuários para legendar e catalogar imagens que atribuem ao tema alimentação saudável. Para isso, construímos uma rede de hashtags de cliques com as hashtags das postagens marcadas com hashtags descritoras do termo "alimentação saudável".

\section{Trabalhos Relacionados}

Muitas pesquisas vêm sendo realizadas nas mídias sociais nas áreas de alimentação e nutrição. Por exemplo, o estudo de Marcon et al. (2018) avaliou como o Instagram é 
usado para descrever e retratar a amamentação e como os usuários compartilham perspectivas e informações sobre esse tema. Esses autores utilizaram hashtags para buscar as postagens e fizeram análise de conteúdo apenas dos textos e das imagens.

Outros estudos também utilizaram hashtags para buscar postagens. Sharma e Choudhury (2015) criaram uma abordagem para extração de informações nutricionais de postagens de alimentos no Instagram utilizando hashtags. E observaram que os usuários tendem a compartilhar fotos e vídeos de alimentos com teor moderado de calorias, enquanto os com alto teor calórico são menos comuns. Gauziski e Maia (2013) fizeram análise dos comentários de 14 imagens do Instagram capturadas por meio da \#grubster para investigar como uma fotografia compartilhada pode transmitir sensações corpóreas, emocionais e cognitivas para as pessoas que as recebem. A busca de postagem utilizando hashtags também é uma estratégia adotada neste estudo.

Além disso, fizemos a análise das hashtags, porém diferentemente de outros estudos, usamos uma rede de hashtags de cliques para isso. Dorsch (2018), por exemplo, analisou hashtags para verificar como os usuários do Instagram marcam suas fotos levando em consideração diferentes tipos de imagem e categorias de hashtag. Esse autor considera que a indexação de imagens e representação do conhecimento pode ser feita por meio da atribuição de hashtags ao conteúdo publicado. Já Türker e Sulak (2017) fizeram uma análise de rede multicamadas de hashtags no Twitter via coocorrência e ligações semânticas. Os autores observaram que a coocorrência de hashtags em tweets é principalmente devido a relações semânticas. A análise da coocorrência das hashtags pode ser feita por meio de uma rede de hashtags de cliques, apresentada neste estudo.

\section{Métodos}

O processo metodológico para realização deste estudo foi sistematizado em sete etapas (Figura 1), as quais serão descritas nas subseções a seguir.

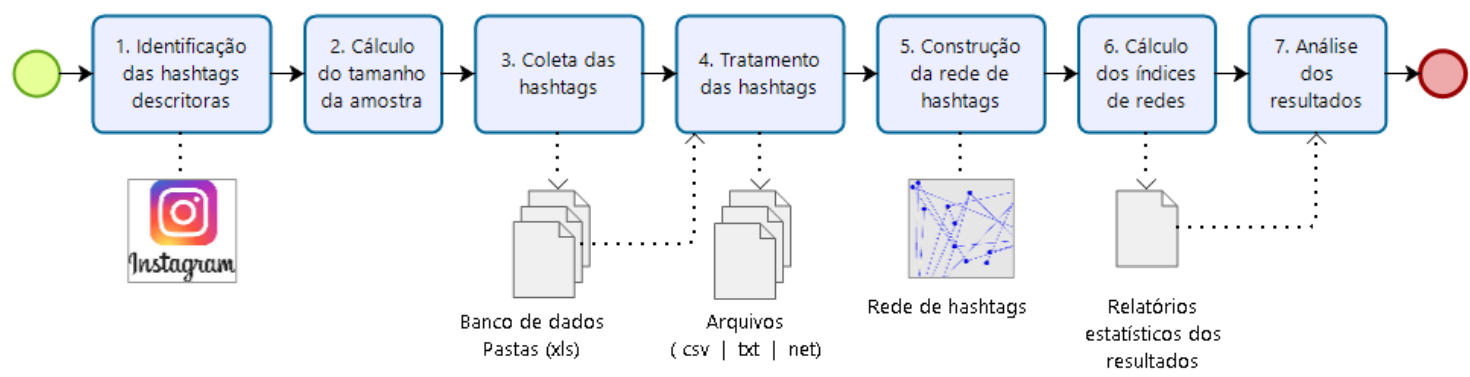

Figura 1. Fluxograma do processo metodológico.

\subsection{Identificação das hashtags descritoras}

Usamos a página oficial do Instagram no diretório de hashtags ${ }^{1}$ para identificar as hashtags representativas do termo "alimentação saudável" mais populares. Três hashtags descritoras foram identificadas e registramos o número total de postagens para cada uma delas em 11 de março de 2019 (i.e., população do estudo): \#alimentacaosaudavel (4.423.026), \#alimentaçãosaudável (1.613.264) e \#alimentaçãosaudavel (881.096).

\footnotetext{
${ }^{1} \mathrm{https}: / /$ www.instagram.com/directory/hashtags/
} 


\subsection{Cálculo do tamanho da amostra}

Para o cálculo do tamanho da amostra foram estabelecidos o nível de confiança de $99 \%$ e erro de 5\%, similares aos usados por Marcon et al. (2018). Assim, para representar o total de postagens presentes no Instagram para cada uma das hashtags descritoras determinamos o número mínimo de 663 postagens por hashtag, ou seja, 1.989 no total.

\subsection{Coleta das hashtags}

Utilizando as hashtags descritoras, capturamos as postagens por meio do Vürku², uma ferramenta paga que permite o download postagens no Instagram por meio de hashtags. O processo de coleta das hashtags está descrito na Figura 2.

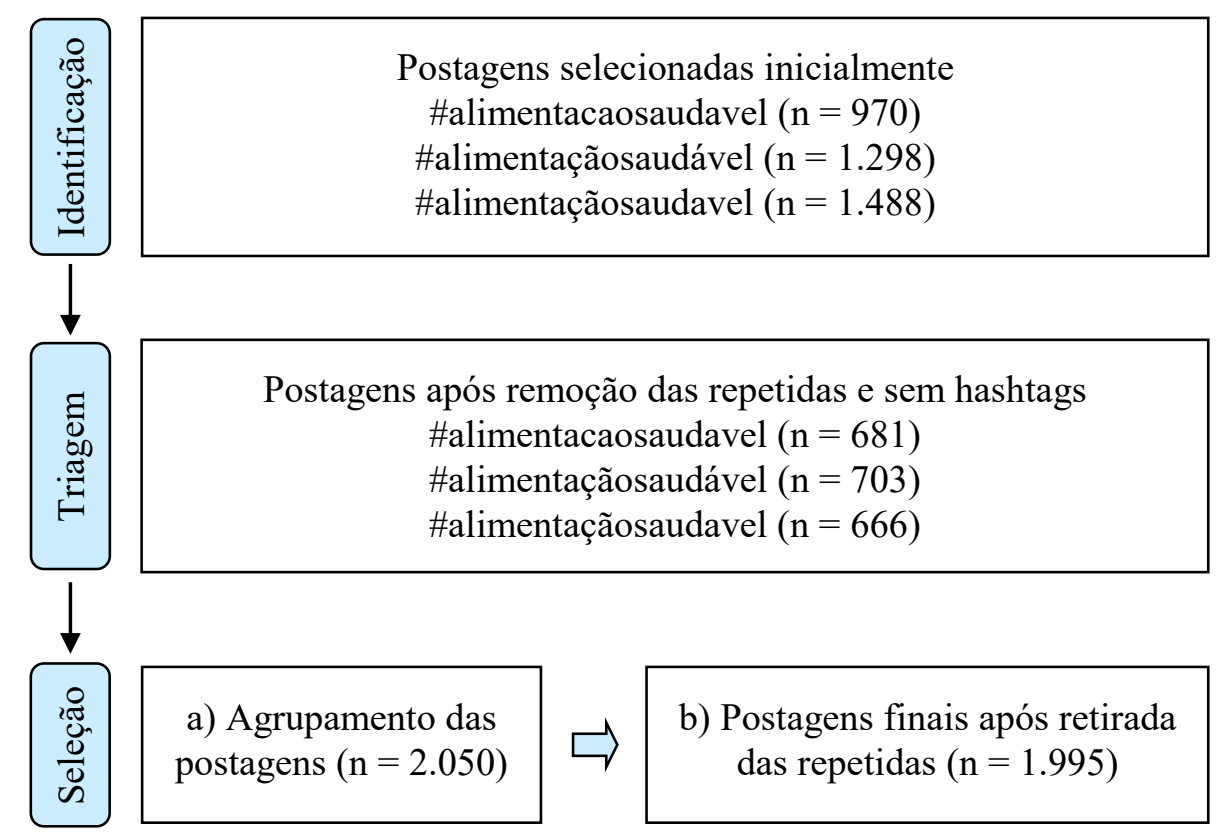

Figura 2. Processo de coleta das hashtags.

Para atingir a amostra para cada hashtag descritora foram necessários três dias sequenciais de coleta, 12, 13 e 14 de março de 2019. Selecionamos um número maior de postagem do que o determinado no cálculo da amostra (Figura 2 - "Identificação"), pois observamos haver postagem sem hashtags e repetidas, que ocorrem quando são compartilhadas mais de uma vez pelo mesmo usuário ou repostadas por usuários diferentes sem alterar quaisquer informações. Não houve critério para seleção das postagens, como popularidade das mesmas em termos de curtidas, senão somente a ocorrência das hashtags descritoras.

$\mathrm{Na}$ "Triagem" foram removidas as postagens repetidas e sem hashtags, e, assim, selecionamos 681 postagens com a \#alimentacaosaudavel, 703 com a \#alimentaçãosaudável e 666 com a \#alimentaçãosaudavel (Figura 2). Em seguida,

\footnotetext{
${ }^{2}$ https://vurku.com
} 
compilamos as postagens das três hashtags descritoras em um só arquivo (Figura 2 "Seleção a") e percebemos a presença de repetição pelo fato de algumas postagens terem sido marcadas com mais de uma das três hashtags descritoras. Após a exclusão de 55 postagens repetidas, a amostra final consistiu em 1.995 postagens (Figura 2 - "Seleção b"), cujas hashtags foram utilizadas na construção da rede.

Após as etapas "Cálculo do tamanho da amostra" e "Coleta das hashtags", obtivemos uma amostra estratificada por hashtags não probabilística. Não foi possível fazer a seleção aleatória das postagens devido a inacessibilidade de toda a população do estudo, que consiste no conjunto de postagens relacionado às hashtags descritoras fornecidas pelo Instagram na data da pesquisa. Por conta disso, não foi possível capturar as postagens da população do estudo, mas sim as que iam sendo postadas a partir da data de início da coleta.

\subsection{Tratamento das hashtags}

O tratamento das hashtags foi realizado conforme as etapas a seguir:

(1) As hashtags foram separadas das postagens manualmente.

(2) As hashtags de cada postagem foram colocadas em uma linha de um arquivo de formato .txt.

(3) O tratamento manual das hashtags consistiu na adaptação e aplicação das regras (R) gerais propostas por Pereira et al. (2011) para redes semânticas de cliques. Com base na referência citada, as regras 3, 6 e 10 não se aplicam a uma rede de hashtags pelo fato de as hashtags já possuírem todas as palavras unidas. Segundo a regra 1, as hashtags de uma postagem consistem em uma sentença. Pela regra 2, os sinais gráficos, como ponto final, ponto-e-vírgula e ponto de interrogação são eliminados, assim como caracteres especiais (e.g., coração ou emoticons), mantendo-se apenas a cerquila (\#). As regras 4, 5 e 8 foram empregadas sem adaptação. Não foi aplicada a regra 7, pois consideramos a escrita das hashtags correta, nem a regra 11, já que é usual usar hashtags em outros idiomas, principalmente em inglês (e.g., \#fitness, \#lowcarb, \#fit, \#healthyfood, \#lifestyle e \#gym). A regra 9 é contemplada no tratamento computacional descrito a seguir.

(4) Após o tratamento manual, foi feito o tratamento computacional das sentenças de hashtags utilizando um conjunto de programas da UNITEX [Paumier 2008] e o conjunto de ferramentas computacionais desenvolvido por Caldeira et al. (2005).

\subsection{Construção da rede de hashtags}

$\mathrm{Na}$ etapa anterior foi gerado um arquivo .net utilizado na construção da rede de hashtags no software Gephi, versão 0.9.2. As redes são representadas por grafos. Segundo Gross e Yellen (1999, p. 2), um grafo $G=(V, E)$ é uma estrutura matemática que consiste em dois conjuntos $V$ (finito e não vazio) e $E$ (relação binária entre $V$ ). Os elementos do conjunto $V$ são os vértices ou nós, e os elementos de $E$ são denominados de arestas. Cada aresta possui um ou dois vértices associados a ela.

Para construção de uma rede semântica de cliques, as unidades de significado de discursos orais ou escritos (e.g., frases, sentenças, títulos, resumos ou falas) são consideradas cliques, subgrafos completos de um grafo $G$ [Fadigas e Pereira 2013]. Cada unidade de significado é uma clique. As cliques se conectam pelos processos de 
justaposição e/ou sobreposição formando a rede de cliques. No processo de justaposição duas cliques são conectadas por apenas um vértice em comum. E no processo de sobreposição, dois ou mais vértices conectam as cliques.

Neste estudo, $G$ é um grafo não dirigido, sendo $V$ o conjunto de hashtags, e $E$ o conjunto de arestas que ligam as hashtags. As hashtags de uma mesma postagem são os vértices de uma clique e as arestas são as conexões entre elas. As hashtags em comum às postagens são os vértices em comum às cliques, que formam a rede de hashtags por sobreposição e justaposição. E consideramos que o conjunto de hashtags sobre um determinado tema representa o discurso sobre esse tema em uma mídia social.

\subsection{Cálculo dos índices de redes}

Os índices para a caracterização e análise da rede de hashtags foram: número de vértices $(n=|V|)$, número de arestas $(m=|E|)$, grau médio $(<k>)$, diâmetro $(D)$, coeficiente de aglomeração médio $(C)$, caminho mínimo médio $(L)$ e densidade $(\Delta)$. Esses índices também foram calculados para o componente gigante da rede de hashtags geral e para a rede aleatória equivalente, a qual foi gerada com o mesmo número de vértices e grau médio do componente gigante e utilizada para caracterização topológica do mesmo. Além desses, utilizamos a centralidade de grau $(C G)$ para medir a importância dos vértices e realizamos a análise de comunidades.

\section{Resultados e Discussão}

Verificamos que a maioria das postagens utilizaram o limite de trinta hashtags estabelecido pelo Instagram. Os usuários das mídias sociais, sejam perfis pessoais ou profissionais, costumam associar suas postagens a várias hashtags com alta popularidade, a fim de que a postagem receba muitas visualizações e viralize. Isso faz com que a postagem fique entre as mais populares, sendo mais facilmente encontrada.

A rede de hashtags geral é constituída por 8.063 vértices e 178.570 arestas (Tabela 1). Em média, as hashtags estão conectadas a aproximadamente 45 outras hashtags $(<k>=44,29)$. Isso sugere que diferentes combinações de hashtags são utilizadas para classificar ou legendar diferentes postagens, e que as mesmas hashtags aparecem em postagens diferentes, haja vista que a média de hashtags por postagem é de 18,39, então para haver conexão média de 45 ocorrem sobreposição ou justaposição de hashtags. Isso está relacionado à necessidade de ampliar o acesso às postagens a fim de que essa receba muitas visualizações.

Tabela 1. Estatísticas básicas da rede geral, do componente gigante da rede geral e da rede aleatória equivalente ao componente gigante.

\begin{tabular}{c|c|c|c|c|c|c|c|c}
\hline Redes & $n=|V|$ & $m=|E|$ & $<k>$ & $D$ & $L$ & $C$ & $\Delta$ & $C M$ \\
\hline Geral & 8.063 & 178.570 & 44,29 & 6 & 2,314 & 0,884 & 0,005 & 25 \\
\hline CG & $\begin{array}{c}8.054 \\
(99,89 \%)\end{array}$ & $\begin{array}{c}178.564 \\
(99,99 \%)\end{array}$ & 44,32 & 6 & 2,314 & 0,884 & 0,006 & 23 \\
\hline RA do CG & 8.054 & 178.005 & 22,101 & 17 & 3,551 & 0,006 & 0,003 & 10 \\
\hline
\end{tabular}

Legenda: $\mathrm{CG}=$ Componente gigante. $\mathrm{RA}$ do $\mathrm{CG}=$ Rede aleatória equivalente ao componente gigante. $\mathrm{CM}=$ Comunidades. Fonte: Autores, 2019. 
O índice coeficiente de aglomeração mede o quanto os vértices vizinhos de um vértice são vizinhos entre si. O coeficiente de aglomeração médio da rede de hashtags é alto $(C=0,884)$. Isso indica que as hashtags vizinhas a uma hashtag estão bem conectadas entre si, já que as hashtags de uma mesma postagem estão todas conectadas entre si, o que condiz com o método de construção de redes de cliques. Também tem a ver com a grande quantidade de hashtags em comum às postagens, o que leva a ocorrência de justaposição e sobreposição. Podemos supor que coeficiente de aglomeração médio alto pode ser um indicativo de menor diversidade de hashtags relacionadas ao tema alimentação saudável nas postagens, ou seja, sempre as mesmas são empregadas nas postagens.

O diâmetro da rede de hashtags é igual a seis $(D=6)$ e três hashtags separam as hashtags $(L=2,314)$. Isso quer dizer que as hashtags são muito próximas, outro indício de que são sempre as mesmas hashtags empregadas quando a postagem está relacionada com o tema alimentação saudável. E também indica justaposição e sobreposição.

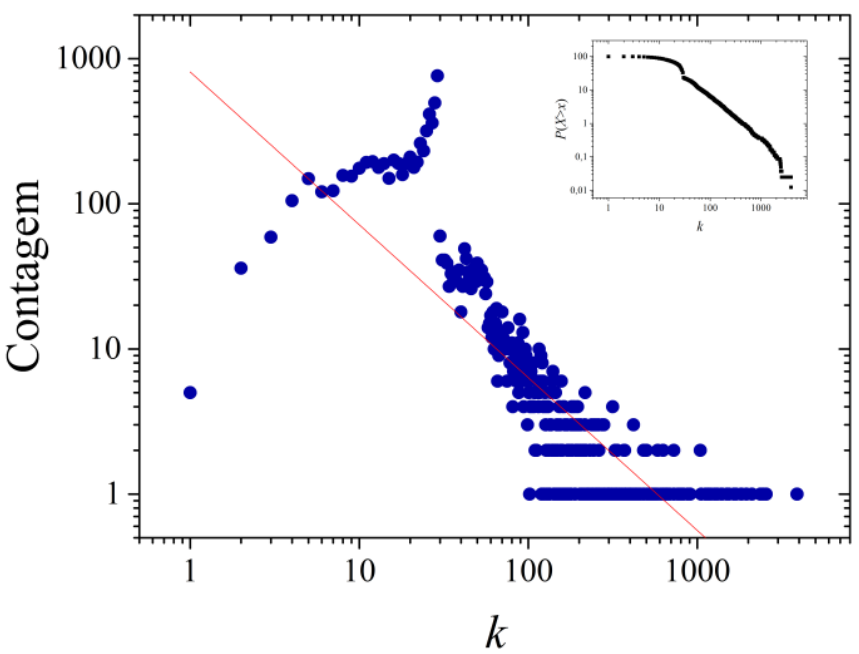

Figura 3. Distribuição de graus do componente gigante da rede de hashtags. No inset, é apresentada a distribuição acumulada. Fonte: Autores, 2019.

Ao realizarmos a caracterização topológica usando o método de Watts e Strogatz (1998) observamos que o componente gigante da rede de hashtags apresenta o fenômeno mundo pequeno, o que indica que as hashtags são muito próximas e muito conectadas, por haver muitas hashtags em comum às postagens relacionadas ao tema alimentação saudável e pela própria característica das cliques. $\mathrm{O}$ fenômeno mundo pequeno em uma rede semântica, a qual é similar a uma rede de hashtags, uma vez que, assim como as palavras, também são imbuídas de significados, aponta para um discurso uniforme em relação ao termo "alimentação saudável" no Instagram na perspectiva da representação do conhecimento pelas hashtags. O caminho mínimo médio é equivalente à mesma propriedade da rede aleatória equivalente $\left(L_{C G}=2,314 ; L_{R A}=3,551\right)$ e o coeficiente de aglomeração médio é muito maior do que o valor da mesma propriedade da rede aleatória equivalente $\left(C_{C G}=0,884 ; C_{R A}=0,006\right)$. Ademais, a distribuição de graus apresenta indícios de uma lei de potência com a $P(k) \sim k^{-\gamma}$, onde $\gamma=1,05 \pm 0,0368$, com $\mathrm{R}^{2}=0,69$ (Figura 3). E isso aponta para a presença de hubs, ou seja, hashtags com alto grau de conexão com outras (e.g., hashtags descritoras, por estarem presentes em todas as postagens, ou seja, em todas as cliques as conectando). 
Para analisar a representação do termo "alimentação saudável" por usuários produtores e consumidores de conteúdo no Instagram identificamos os principais vértices da rede em termos de centralização de grau. E para verificar se essa representação está em consonância com o conceito de alimentação adequada e saudável do Guia Alimentar para a População Brasileira (Brasil 2014) identificamos nas comunidades categorias temáticas relacionadas aos princípios básicos desse conceito.

Considerando a análise de redes sociais, investigamos a importância dos vértices por meio dos índices de centralidade de grau, as hashtags mais associadas a outras hashtags (Figura 3). As dez hashtags de maior grau, em ordem decrescente, são: \#saude, \#dieta, \#fitness, \#vidasaudavel, \#comidadeverdade, \#emagrecer, \#emagrecimento, \#nutrição, \#saúde e \#reeducaçãoalimentar. As hashtags \#alimentaçãosaudável, \#alimentacaosaudavel e \#alimentaçãosaudavel foram excluídas da análise das 10 mais importantes em relação ao grau por terem sido empregadas como descritores de busca.

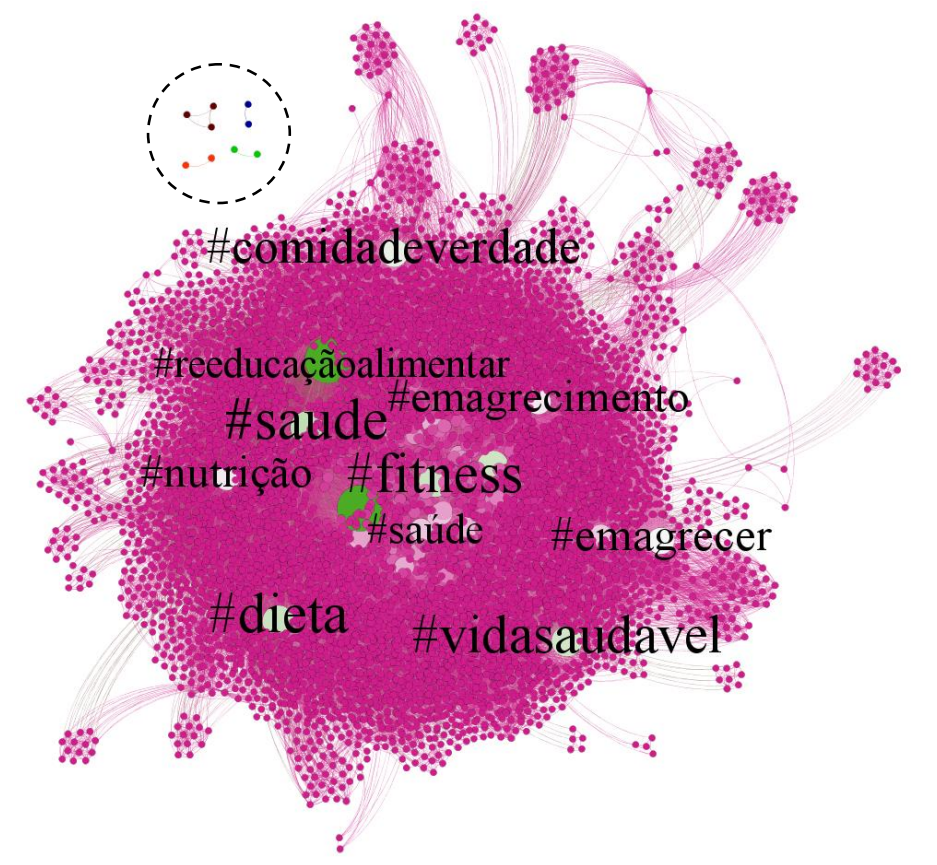

Figura 3. Rede de hashtags sobre alimentação saudável. No componente gigante os vértices estão coloridos do magenta (menor grau) ao verde (maior grau), passando pelo branco (graus intermediários). Os outros quatro componentes estão circulados e em outras cores. Fonte: Autores, 2019.

Essas hashtags representam os temas mais conectados dentro do discurso sobre alimentação saudável no Instagram no momento investigado. Os vértices \#dieta, \#fitness, \#emagrecer, \#emagrecimento e \#nutrição ressaltam a importância que é dada ao aspecto biológico (i.e., atendimento as necessidades nutricionais) da alimentação adequada e saudável no discurso das mídias sociais em detrimento de outros. Além disso, esses vértices refletem a existência de forte associação entre saúde e estética no discurso sobre alimentação saudável no Instagram, a qual está relacionada à busca do corpo culturalmente construído e difundido nas mídias sociais. A preocupação incessante com a saúde relacionada com os cuidados com o corpo estabelece a todo momento a conexão entre saúde e estética, denotando que saúde é estar em forma [Costa, Carvalho e Prado 2013]. 
$\mathrm{Na}$ rede de hashtags, foram observadas 25 comunidades com o índice de modularidade igual a 0,137 (Figura 4). As comunidades na rede de hashtags formam grupos de hashtags bem integrados ou correlacionados. Fizemos a análise das 10 comunidades que apresentaram os maiores percentuais de vértices (maior que 4\%), totalizando $80 \%$ dos vértices da rede, e que continham as três hashtags descritoras. Nas comunidades destacamos as 10 hashtags com maiores centralidades de grau (Figura 4), apresentadas nos parágrafos a seguir em ordem decrescente. E, dentre essas, destacamos em negrito a maioria com correlação semântica, a fim de identificar categorias temáticas relacionadas ao conceito de alimentação adequada e saudável.

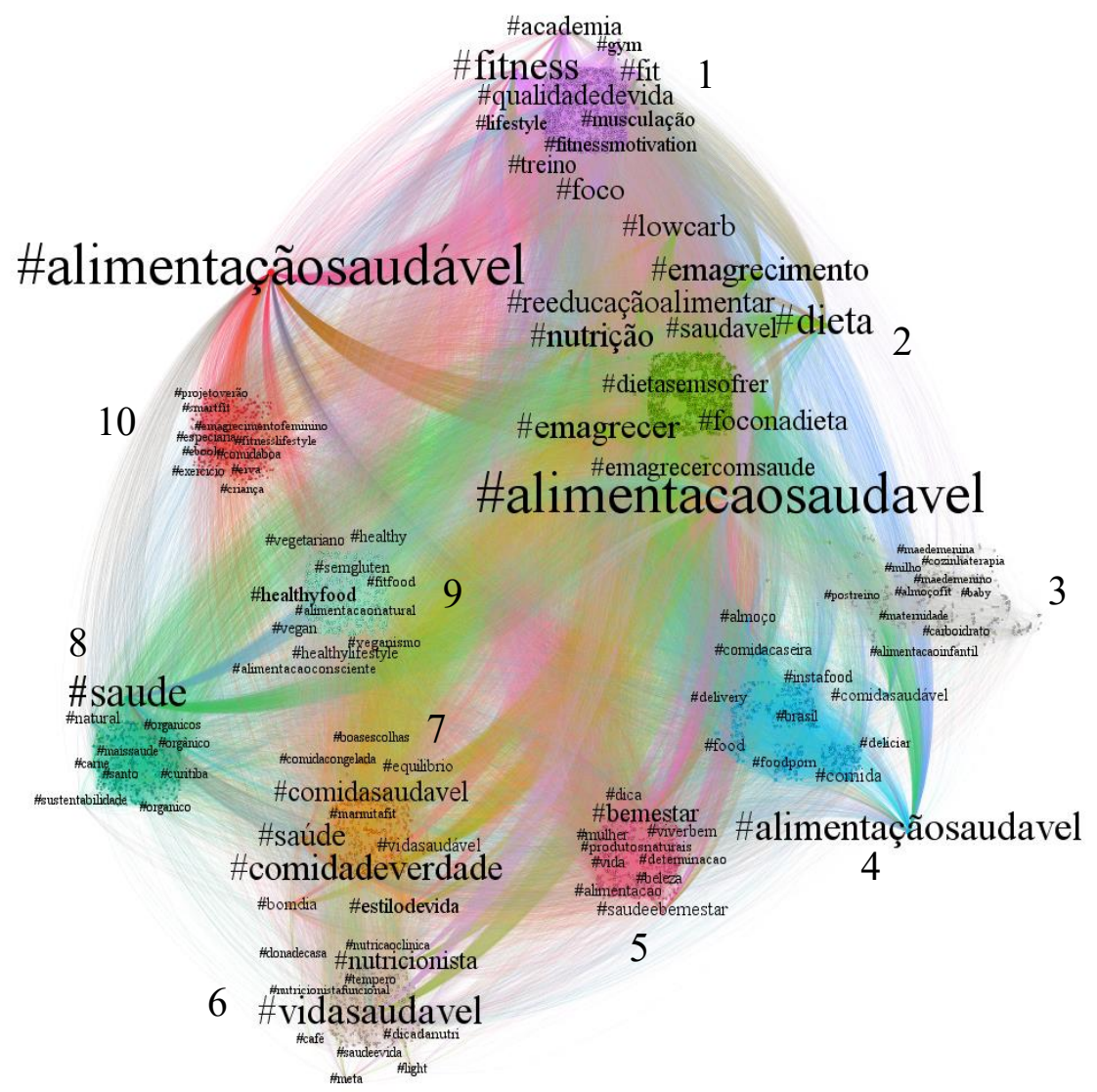

Figura 4. Distribuição espacial de dez comunidades da rede de hashtag. 0 tamanho dos rótulos é proporcional ao grau dos vértices. Fonte: Autores, 2019.

Na comunidade 1, os principais vértices em termo de grau são \#fitness, \#foco, \#fit, \#qualidadedevida, \#academia, \#treino, \#musculação, \#fitnessmotivation, \#lifestyle e \#gym (Figura 4). Por essa comunidade agrupar a maior parte dos vértices $(18,74 \%)$, isso indica que a temática "fitness" predomina dentre as demais no discurso sobre alimentação saudável no Instagram. Esse achado reflete a busca incessante pelo modelo cultural vigente de corpo ideal (magro e forte) difundido pelas mídias sociais. Isso constribui para a insatisfação crônica com o corpo, levando a busca por exercícios físicos estenuantes (reforçada pela \#foco) e por dietas muito restritivas. A presença de outra comunidade com essa mesma temática reforça ainda mais esse discurso, no caso a comunidade 10 (4,22\%): \#comidaboa, \#erxecício, \#especiaria, \#fitnesslifestyle, \#emagrecimentofeminino, \#projetoverão, \#ebook, \#erva, \# smartfit e \# praticar. A presença de outra comunidade sobre a temática "fitness". 
A comunidade 2 agrupa 17,92\% dos vértices e as hashtags como maior grau estão dentro da temática "emagrecimento": \#dieta, \#emagrecer, \#emagrecimento, \#nutrição, \#reeducaçãoalimentar, \#lowcarb, \#foconadieta, \#saudavel, \#dietasemsofrer e \#emagrecercomsaude. $O$ desejo de perda rápida de peso para alcançar o ideal de magreza veiculado pelas mídias estimula a adesão às dietas da moda e tem corroborado para o aumento da incidência de transtornos alimentares. As dietas da moda sempre marcaram presença nas revistas voltadas para o público feminino e agora são difundidas nas mídias sociais [Costa, Carvalho e Prado 2013].

A comunidade 3 (4,33\%) representa a temática "nutrição materno-infantil", com o agrupamento das hashtags: \#alimentacaoinfantil, \#carboidrato, \#maternidade, \#postreino, \#maedemenino, \#cozinhaterapia, \#almoçofit, \#maedemenina, \#baby e \#milho. Marcon et al. (2018) observaram que o Instagram está sendo usado para exibir e compartilhar publicamente diversos conteúdos relacionados à amamentação e para criar redes de apoio que permitam que mães compartilhem experiências, o que corrobora com o achado neste estudo de uma comunidade sobre a temática nutrição materno-infantil.

A comunidade 4 com 6,96\% dos vértices agrupa as hashtags \#comida, \#food, \#comidasaudável, \#instafood, \#almoço, \#brasil, \#comidacaseira, \#delivery, \#foodporn e \#deliciar, as quais representam a temática "comida". A comunidade 7 $(6,15 \%)$ também possui essa temática, mas de forma menos explícita: \#comidadeverdade, \#saúde, \#comidasaudavel, \#estilodevida, \#vidasaudável, \#bomdia, \#equilibrio, \#marmitafit, \#boasescolhas e \#comidacongelada. Assim como a comunidade 4 também está na temática "comida". Essas comunidades se aproximam dos aspectos culturais, sociais e hedônicos relacionados a alimentação adequada e saudável. No Instagram é comum os usuários mostrarem as fotos do pratos que comem e preparam para que sejam avaliados positivamentes e ganhem capital social nas mídias.

A comunidade 5 (5,71\%) agrupa as hashtags \#bemestar, \#saudeebemestar, \#alimentacao, \#dica, \#viverbem, \#vida, \#mulher, \#beleza, \#determinacao e \#produtosnaturais, relacionadas a temática "bem-estar". A temática não é muito explícita, pois apenas quatro hashtagas apresentaram essa correlação semântica. $O$ termo "bem-estar" relaciona-se com a promoção da saúde integral dos indivíduos, o que necessariamente envolve todos os aspectos da alimentação adequada e saudável.

A comunidade 6 (4,39\%) agrupa hashtags (\#vidasaudavel, \#nutricionista, \#dicadanutri, \#meta, \#light, \#nutricaoclinica, \#donadecasa, \#saudeevida, \#café e \#tempero) relacionadas a temática "nutricionista". Essse profissionais de saúde têm investido em marketing e publicidade nas mídias sociais para divulgar seu trabalho e ganhar vizibilidade dentro e fora das mídias.

A comunidade 8 (4,94\%) está relacionada temática da "sustentabilidade": \#saude, \#natural, \#organicos, \#curitiba, \#carne, \#orgânico, \#organico, \#maissaude, \#sustentabilidade e \#santo. Diferente das outras comunidades que expressam temáticas relacionadas ao aspecto biológico da alimentação adequada e saudável, a comunidade 7 enfatiza a premissa de que a alimentação saudável deve ser baseada em práticas produtivas adequadas e sustentáveis, como a produção de alimentos orgânicos e oriundos da agrucultura familiar. Assim como a comunidade $9(6,85 \%)$ sobre a temática "vegetarianismo", que reflete o interesse por esse padrão de consumo alimentar na contemporaneidade. As principais hashtags dessa comunidade são: \#healthyfood, 
\#healthy, \#healthylifestyle, \#semgluten, \#vegan, \#vegetariano, \#fitfood, \#alimentacaoconsciente, \#veganismo, \#alimentacaonatural.

Observamos neste estudo que a maioria das comunidades analisadas apresentaram temáticas relacionadas aos aspectos biológicos da alimentação adequada e saudável, ou seja, atendimento às necessidades nutricionais, e a questões relacionadas a estética do corpo. Outras comunidades que agrupam um menos número de vertices também possuem hashtags que representam os aspectos sociais, culturais e hedônimos do conceito de alimentação adequada e saudável exporado neste estudo.

\section{Considerações Finais}

Neste estudo foi possível analisar como o tema alimentação saudável tem sido representado em uma mídia social e a sua consonância com o conceito de alimentação adequada e saudável do Guia Alimentar para a População Brasileira [Brasil 2014] por meio de uma rede de hashtags de cliques.

As hashtags correlacionadas ao tema alimentação saudável estão conectadas em média a 45 outras hashtags indicando que há variedade de hashtags relacionadas ao tema. Também observamos alta aglomeração na rede de hashtags, o que pode indicar que as mesmas hashtags são citadas em diferentes postagens, levando aos processos de justaposição e sobreposição entre as cliques.

Os vértices mais importantes em termos de centralidade de grau refletem a ênfase dada ao aspecto biológico da alimentação adequada e saudável em detrimento de outros e a postura midiática de culto ao corpo magro e forte, o que também foi observado nas comunidades analisadas. As temáticas identificadas nessas comunidades representam o discurso sobre o tema alimentação saudável na mídia social Instagram, a saber: "fitness", "emagrecimento", “comida", "bem-estar", "vegetarianismo", "sustentabilidade", "nutricionista", "nutrição materno-infantil". Porém, a análise isolada das hashtags não é suficiente para tirar conclusões sobre o discurso proferido, sendo necessário associá-la a análise das imagens, textos, perfis, comentários e outros elementos das postagens.

Este estudo contribui como um método para prospecção do discurso sobre alimentação saudável em mídias sociais por meio da análise da hashtags utilizadas para classificar as postagens. Porém, para ter mais subsídios e tirar conclusões mais acertivas sobre os discursos proferidos pelos usuários de mídias sociais sugerimos ampliar a análise de hashtags para uma análise que considere os perfis, imagens, textos e hashtags. Ademais, identificamos a necessidade de ferramentas de livre acesso para a coleta de dados. Por isso, tornam-se necessários estudos sobre a difusão de informações em mídias sociais, desenvolvimento de ferramentas que subsidiem a coleta, tratamento e análise dos dados, e que reflitam sobre o uso das mídias sociais na promoção da saúde.

\section{Referências}

AFP. (2018) “Instagram superou 1 bilhão de usuários ativos”. Exame. Disponível em: https://exame.abril.com.br/tecnologia/instagram-superou-1-bilhao-de-usuariosativos/. Acesso em: 2019, março. 
Brasil. Ministério da Saúde. (2014) "Guia Alimentar para a População Brasileira". Ministério da Saúde, Secretaria de Atenção à Saúde, Departamento de Atenção Básica. - 2. ed. - Brasília: Ministério da Saúde.

Brasil. Secretaria Especial de Comunicação Social. (2016) "Pesquisa brasileira de mídia 2016: hábitos de consumo de mídia pela população brasileira”. Brasília: Secom.

Caldeira, S. M. G. (2005) "Caracterização da rede de signos linguísticos: Um modelo baseado no aparelho psíquico de Freud”. Mestrado Interdisciplinar em Modelagem Computacional, Fundação Visconde de Cairu, Salvador.

Costa, M. F.; Carvalho, M. C. V. S.; Prado, S. D. (2013) "Dietas da Moda: um processo incessante e ininterrupto". In: Alimentação, consumo e cultura. $1^{a}$ edição. (Org.): Francisco Romão Ferreira; Ricardo Ferreira Freitas; Shirley Donizete Prado. Série Sabor Metrópole, volume 1, p. 103-121.

Dorsch, I. (2018). "Content description on a mobile image sharing service: Hashtags on instagram." Journal of Information Science Theory and Practice 6(2): 46-61.

Fadigas, I. S. e Pereira, H. B. B. (2013) "A network approach based on cliques". In Physica A: Statistical Mechanics and its Applications, 392(10):2576-2587.

Kuwae, C. A. et al. (2015) Mercado do saudável: consumo, alimentação e saúde. In: Alimentação e Consumo de Tecnologias $1^{\mathrm{a}}$ ed. (Org.): Shirley D. Prado, Fabiana B. Kraemer, Cristiane M. Seixas e Ricardo Ferreira Freitas. Curitiba. (1):349-362.

Gauziski, D. e Maia, A. (2013) "Produção de presença em imagens Gastronômicas no Instagram". In: Alimentação, consumo e cultura. $1^{\text {a }}$ edição. (Org.): Francisco R. Ferreira; Ricardo F. Freitas; Shirley D. Prado. Sabor Metrópole, vol. I, p. 193-203.

Gross, J. T. e Yellen, J. (1999) "Graph. Theory and its Applications”. Boca Raton: CRC Press.

Marcon, A., Bieber, M. e Azad, M. (2018) "Protecting, promoting, and supporting breastfeeding on Instagram", In Matern Child Nutr, 15:12658. Disponível em: https://onlinelibrary.wiley.com/doi/epdf/10.1111/mcn.12658

Paumier, S. (2008) “User Manual”, UNITEX 2.0. Université Paris-Est Marne-la-Vallée, Disponível em: http://unitexgramlab.org/releases/2.0/man/Unitex-GramLab-2.0usermanual-en.pdf. Acesso em: 2019, março.

Pereira, H. B. B. et al. (2011) "Semantic networks based on titles of scientific papers". In Physica A: Statistical Mechanics and its Applications, 390(6):1192-1197.

Sharma, S. S. e Choudhury M. (2015) "Measuring and Characterizing Nutritional Information of Food and Ingestion Content in Instagram". In: Proceedings of the 24th International Conference on World Wide Web. Florença, Itália.

Türker, I. e Sulak, E. E. (2017) "A multilayer network analysis of hashtags in twitter via co-occurrence and semantic links". In International J. of Modern Physics B, (31).

Watts, D. J. e Strogatz, S. H. (1998) "Collective dynamics of small-world networks". In Nature, 393(4):440-442. 